\title{
18. On the Distribution of the Zero Points of Sections of a Power Series.
}

By Masatsugu Tsus.

(Received December 10, 1924.)

1.

By Jentzch's theorem we know that every point on the convergence circle is a limiting point of the zero point of sections of a power series and Hurwitz's theorem teaches us that inside the convergence circle the limiting point is the zero of the function defined by the power series. The purpose of this paper is to investigate the distribution of the limiting points outside the convergence circle ander a certain broad assumption.

1. Let the power series be

$$
F(x)=a_{0}+a_{1} x+a_{2} x^{2}+\cdots+a_{n} x^{n}+\cdots
$$

and any root of the equation

$$
a_{0}+a_{1} x+a_{2} x^{2}+\cdots+a_{n} x^{n}=0
$$

be denoted by $x_{n}$.

Theorem I. If for non-vanishing coefficients $a_{n}$ of $(1)$

$$
\begin{aligned}
& \varlimsup_{n=\infty}\left|\frac{a_{n}}{n^{\lambda} \log ^{\lambda_{0}} n \log _{1}^{{ }^{\lambda}} n \ldots \log _{\nu}{ }^{\lambda \nu} n}\right|=K<\infty \\
& \lim _{n=\infty}\left|\frac{a_{n}}{n^{\lambda} \log ^{\lambda_{0} n} \log _{1}{ }^{{ }_{1}} n \cdots \log _{\nu}{ }^{\lambda \nu} n}\right|=L>0 .
\end{aligned}
$$

where $\log _{1} n=\log (\log n)$ etc. and $\lambda \lambda_{0} \lambda_{1} \ldots \lambda_{\nu}$ are any real numbers, then

$$
\overline{\lim }_{n=\infty}\left|x_{n}\right| \leqq e^{\frac{K}{L}}
$$

Remark. The zero points are bounded and the limiting points lie in the circle

$$
|x|=e^{\frac{K}{L}}
$$

2. We first prove two lemmas:

Lemma I. When $r \geqq r_{o}>1, \Phi(t)=r^{t} t^{\lambda} \log ^{\lambda \rho} t \log _{1}{ }^{{ }} 1 t \cdots \log _{v}{ }^{\lambda \nu} t$ becomes increasing for $t \geqq n_{o}$, where $n_{o}$ is independent of $r$. Proof. Putting

$$
f(t)=t^{\lambda} \log ^{\lambda \rho} t \cdots \log _{\nu}^{\lambda v} t
$$


We get

$$
t f^{\prime}(t)=f(t) \psi(t)
$$

where

$$
\psi(t)=\lambda+\frac{\lambda_{o}}{\log t}+\cdots+\frac{\lambda_{v}}{\log t \cdot \log _{v} t}
$$

Sinee $\Phi(t)=r^{t} f(t)$, we get from $(3)$

$$
\Phi^{\prime}(t)=r^{t} f^{\prime}(t)+r^{t} f(t) \log r=r^{t} f(t)\left[\log r+\frac{\psi(t)}{t}\right]
$$

Now since $\lim _{t=\infty} \frac{\psi(t)}{t}=0$, we can find $\mathrm{n}_{o}$ so that for $t \geqq n_{o}$

$$
\left|\frac{\psi(t)}{t}\right|<\varepsilon_{1}
$$

where $\epsilon_{1}$ is a small quantity.

If we determine $\varepsilon_{1}$ so that $\log r_{3}>\epsilon_{1}$ or $r_{0}>e^{\varepsilon_{1}}$

then we have $\Phi^{\prime}(t)>0$ uniformly for $r \geqq r_{0}$. Hence the lemma is proved.

Lemma. II. Wheu $\alpha$ is given, we can find $n_{0}$ and $\bar{r}_{0}$ such that for $n_{2} \geqq n_{0}$,

$$
\int_{n_{2}}^{n} \Phi(t) d t<\frac{1}{\mu} \Phi(n)
$$

uniformly for $r \geqq \check{r}_{0}>1$.

Proof.

By integrating

$$
\Phi^{\prime}(t)=\Phi(t)\left[\log r+\frac{\psi(t)}{t}\right]
$$

we get

$$
\int_{n_{2}}^{n} \Phi(t)\left[\log r+\frac{\psi(t)}{t}\right] d t=[\Phi(t)]_{n_{2}}^{n}
$$

Since for a large value of $t, \Phi(t)$ is positive, we get

$$
\int_{n_{2}}^{n} \Phi(t)\left[\log r+\frac{\psi(t)}{t}\right] d t<\Phi(n)
$$

Now we determine $\bar{r}_{0}$ so that

$$
\log \bar{r}_{0}>\mu+s_{1} \quad \text { or } \quad \bar{r}_{0}>e^{\mu+\varepsilon_{1}}
$$

Then from (5), we see that

$$
\log r+\frac{\psi(t)}{t}>\mu
$$


for $t \geqq n_{0}$ and $r \geqq \bar{r}_{0}$.

Consequently from (6), we get,

$$
\begin{gathered}
\mu \int_{n_{2}}^{n} \Phi(t) d t<\int_{n_{2}}^{n} \Phi(t)\left[\log r+\frac{\psi(t)}{t}\right] d t<\Phi(n) \\
\int_{n_{2}}^{n} \Phi(t) d t<\frac{1}{\mu} \Phi(n)
\end{gathered}
$$

for $n_{2} \geqq n_{0}$ and $r \geqq \bar{r}_{0}$

3. Now from the hypotinesis of the theorem I, we can put for $n \geqq n_{1}$

or

$$
\begin{aligned}
& L-\varepsilon \leqq\left|\frac{a_{n}}{n^{\lambda} \log ^{\lambda o} n \ldots \log _{\nu}^{\lambda v} n}\right| \leqq K+\varepsilon \\
& L-\varepsilon \leqq\left|\frac{a_{n}}{f(n)}\right| \leqq K+\varepsilon
\end{aligned}
$$

where $e$ is a small quantity.

Putting $|x|=r$, we get,

$$
\begin{array}{r}
\left|a_{n_{1}} x^{n_{1}}+\cdots+a_{n-1} x^{n-1}\right| \leqq\left|a_{n_{1}} x^{n_{1}}\right|+\cdots+\left|a_{n-1} x^{n-1}\right| \leqq \\
(K+\varepsilon)\left[f\left(n_{1}\right) r^{n_{1}}+\cdots+f(n-1) r^{n-1}\right]=(K+\varepsilon)\left[\Phi\left(n_{1}\right)+\cdots+\Phi(n-1)\right]
\end{array}
$$

Take $n_{1}$ greater than $n_{3}$ of. Lemma I, and $r \geqq r_{o}$, then, since by Lemma I, $\Phi(t)$ is increasing hence we have,

$$
\Phi\left(n_{1}\right)+\cdots+\Phi(n-1) \leqq \int_{n_{1}}^{n} \Phi(t) d t
$$

Take $n_{1}$ greater than $n_{0}$ of Lemma II and $r \stackrel{\equiv}{=} \bar{r}_{o}$, then,

$$
\int_{n_{1}}^{n} \Phi(t) d l<\frac{1}{\mu} \Phi(n)
$$

Hence we have

$$
\left|a_{n_{1}} x^{n_{1}}+\cdots+a_{n-1} x^{n-1}\right|<\frac{K+\varepsilon}{\mu} \Phi(n)
$$

Putting

$$
M=\left|a_{0}\right|+\cdots+\left|a_{n_{1}-1}\right|
$$

we get

$$
\left|a_{0}+a_{1} x+\cdots+a_{n_{1}-1} x^{n_{1}-1}\right|<M r^{n_{1}}
$$

Therefore 


$$
\begin{aligned}
\mid a_{0}+\cdots+a_{n-1} & x^{n-1} \mid<M r^{n_{1}}+\frac{K+\varepsilon}{\mu} \Phi(n) \\
& =\frac{K+\varepsilon}{\mu} \Phi(n) \cdot\left[1+\frac{M \mu}{(K+\varepsilon)} \frac{r^{n_{1}}}{\Phi(n)}\right]
\end{aligned}
$$

We will show that the second term in the bracket in (12) can be made very small.

$$
\text { For } \begin{aligned}
r \geqq \vec{r}_{0} \\
\qquad \frac{r^{n_{I}}}{\Phi(n)}=\frac{1}{r^{n-n_{1}} n^{\lambda} \log _{\lambda_{0}} n \cdots \cdot \log _{\nu}^{\lambda \nu} n} \leqq \frac{1}{\bar{r}^{n-n_{1}} n^{\lambda} \cdots \log _{\nu}^{\lambda \nu} n}
\end{aligned}
$$

Since $\lim _{n=\infty} \frac{1}{\vec{r}_{0}^{n-n_{1}} n^{\lambda} \ldots \log _{v}^{\lambda \nu} n}=0$, we can determine $n_{3}$ so large, that for $n \geqq n_{3}$ and $r \geqq \vec{r}_{0}$ we have,

Hence

$$
\left|\frac{\not \pi r^{n_{1}}}{(K+\varepsilon) \Phi(n)}\right|<\epsilon_{2}
$$

$$
\left|a_{0}+a_{1} x+\cdots+a_{n-1} x^{n-1}\right|<\frac{R+\epsilon}{\mu} \Phi(n)\left[1+s_{2} \mu\right]
$$

If we determine $\mu$, not specified till now, so that

$$
L-\varepsilon \geqq \frac{K+\varepsilon}{\mu}\left(1+\varepsilon_{2} \mu\right) \text { or } \mu \geqq \frac{K+\varepsilon}{L-\hat{\varepsilon}-\varepsilon_{2}(K+\varepsilon)},
$$

Then

$$
\begin{aligned}
\left|a_{n} x^{n}\right| \geqq(L-\varepsilon) f(n) r^{n} \geqq \frac{K+\varepsilon}{\beta^{\prime}}\left(\mathbf{I}+\varepsilon_{2}, \mu\right) \Phi(n)> \\
\quad\left|a_{n}+a_{1} x+\cdots+a_{n-1} x^{n-1}\right|
\end{aligned}
$$

Hence for $r \geqq \bar{r}_{0}$ and $n \geqq n_{3}$

$$
a_{0}+a_{1} x+\cdots+a_{n} x^{n} \neq 0
$$

consequently we get,

$$
\varlimsup_{n=\infty}\left|x_{n}\right| \leqq \bar{r}_{0}
$$

or from (7)

$$
\varlimsup_{n=\infty}\left|x_{n}\right| \leqq e^{n+\epsilon_{1}}
$$

Since $\varepsilon_{1} \varepsilon_{1}$ and $\varepsilon_{2}$ can be taken very small, we get from (14) and (15) 


$$
\overline{\lim }_{n=\infty}\left|x_{n}\right| \leqq e^{\frac{K}{L}}
$$

whicin proves tise theorem.

Remank. When the coefficients $a_{n}$ of the power series (1) satisfy the condition of Theorem I., then $\lim _{n=\infty}\left|a_{n}\right|^{\frac{1}{n}}=1$, i. e., the radius of convergence of tise power series is 1 .

\section{2.}

4. We will prove tiogt if $\lim _{n=\infty} \frac{a_{i n}}{n^{k}}=A=0$, then there are no limiting points outside the unit-circle, where $t h$ is any real number. First we will prove three lemmas.

Lemma 1 . In the domain $D$ definet by $1<r_{0} \leqq|x| \leqq r_{1}$ there exists a positive constant $A$ which is independent of $x$ and $n$, such that

$$
\left|\frac{x}{1}+\frac{x^{2}}{2^{k}}+\cdots+\frac{x^{n}}{n^{k}}\right|>A\left|\frac{x^{n}}{n^{k}}\right|
$$

for $n \geqq n_{0}$, where $k$ is any real number.

We will distinguisin the two cases, according as $k>0$ or $k \leqq 0$

5. Proof of the lemma for $k>0$.

Putting $\quad \phi(t)=\frac{x}{1}+\frac{x^{2}}{2^{k}}+\cdots+\frac{x^{i n}}{n^{k}}$

we have

$$
\begin{aligned}
\varphi(t)=\frac{1}{\Gamma(k)} \int_{0}^{1} \frac{1}{t}\left(\log \frac{1}{t}\right)^{k-1}(t x+ & \left.\cdots+t^{n} x^{n}\right) d t \\
& =\frac{1}{\Gamma(k)} \int_{0}^{1} \frac{1}{t}\left(\log \frac{1}{t}\right)^{k-1} \frac{x^{n+1} t^{n+1}-x t}{x t-1} d t \cdots \cdots
\end{aligned}
$$

Since $x$ lies in the domain $D, \frac{1}{|x|} \leqq \frac{1}{r_{0}}<1$

When we put $t_{0}=\frac{1}{|x|}$ and $\tau_{0}=\frac{1}{r_{0}}$, then $t_{0}=\frac{1}{|x|} \leqq \frac{1}{r_{0}}=\tau_{0}<1$.

Denoting by $\varepsilon$ a small quantity, to be specified later on, we decompose the integral (2) into two parts:

$$
\int_{0}^{1}=\int_{0}^{t_{0}+\epsilon}+\int_{t_{0}+\epsilon}^{1}
$$


1.14

M. TSUJT

which we estimate

a)

$$
\begin{aligned}
& \left|\int_{0}^{t_{0}+\varepsilon}\left(\log \frac{1}{t}\right)^{k-1}\left(x+x^{2} t+\cdots+x^{n} t^{n-1}\right) d t\right| \\
& \quad \leqq \int_{0}^{t_{0}+\varepsilon}\left(\log \frac{1}{t}\right)^{k-1}\left(|x|+|x|^{2} t+\cdots+|x|^{n} t^{n-1}\right) d t \\
& \quad<\left(|x|+|x|^{2}\left(t_{0}+\varepsilon\right)+\cdots+|x|^{n}\left(t_{0}+\varepsilon\right)\right)^{n-1} \int_{0}^{t_{0}+\varepsilon}\left(\log \frac{1}{t}\right)^{k-1} d t \\
& \quad=|x|\left(1+(1+\varepsilon|x|)+\cdots+(1+\varepsilon|x|)^{n-1}\right) \int_{0}^{t_{0}+\varepsilon}\left(\log \frac{1}{t}\right)^{k-1} d t \\
& \quad<n|x|(1+\varepsilon|x|)^{n-1} \int_{0}^{1}\left(\log \frac{1}{t}\right)^{k-1} d t=n|x|(1+\varepsilon|x|)^{n-1} \Gamma^{\prime}(k)
\end{aligned}
$$

Hence

$$
\left|\int_{0}^{t_{0}+\varepsilon} \frac{1}{t}\left(\log \frac{1}{t}\right)^{k-1} \frac{x^{n+1} t^{n+1}-x t}{x t-1} d t\right|<n|x|(1+\varepsilon|x|)^{n-1} \Gamma^{\prime}(k)
$$

b)

$$
\begin{aligned}
& \left|\int_{t_{0}+\epsilon}^{1} \frac{1}{t}\left(\log \frac{1}{t}\right)^{k-1} \frac{x^{n+1} t^{n+1}-x t}{x t-1} d t\right| \geqq\left|\int_{t_{0}+\epsilon}^{1}\left(\log \frac{1}{t}\right)^{k-1} \frac{x^{n+1} t^{n}}{x t-1} d t\right| \\
& -\left|\int_{t_{0}+\epsilon}^{1}\left(\log \frac{1}{t}\right)^{k-1} \frac{x}{x t-1} d t\right| \\
& \left|\int_{t_{0}+\varepsilon}^{1}\left(\log \frac{1}{t}\right)^{k-1} \frac{x}{x t-1} d t\right| \leqq|x| \int_{t_{0}+\epsilon}^{1}\left(\log \frac{1}{t}\right)^{k-1} \frac{d t}{|x t|-1} \\
& <\frac{|x|}{|x|\left(t_{0}+\varepsilon\right)-1} \int_{t_{0}+\varepsilon}^{1}\left(\log \frac{1}{t}\right)^{k-1} d t<\frac{|x|}{|x|\left(t_{0}+\varepsilon\right)-1} \int_{0}^{1}\left(\log \frac{1}{t}\right)^{k-1} d t \\
& =\frac{1}{\varepsilon} \int_{0}^{1}\left(\log \frac{1}{t}\right)^{k-1} d t=\frac{\Gamma(k)}{\varepsilon}
\end{aligned}
$$

Hence

$$
\left|\int_{t_{0}+\varepsilon}^{1}\left(\log \frac{1}{t}\right)^{k-1} \frac{x}{x t-1} d t\right|<\frac{\Gamma(k)}{\varepsilon}
$$

And

$$
\begin{gathered}
\left|\int_{t_{0}+\epsilon}^{1}\left(\log \frac{1}{t}\right)^{k-1} \frac{x^{n+1} t^{n}}{x t-1} d t\right|=|x|^{n+1}\left|\int_{t_{0}+\epsilon}^{1}\left(\log \frac{1}{t}\right)^{k-1} \frac{t^{n}}{x t-1} d t\right|= \\
|x|^{n+1} \sqrt{\left[\int_{t_{0}+\epsilon}^{1}\left(\log \frac{1}{t}\right)^{k-1} \frac{t^{n}(\xi t-1)}{(\xi t-1)^{2}+\eta^{2} t^{2}} d t\right]^{2}+\left[\int_{t_{0}+\epsilon}^{1} \cdot\left(\log \frac{1}{t}\right)^{k-1} \frac{\eta t^{n+1}}{(\xi t-1)^{2}+\eta^{2} t^{2}} d t\right]}
\end{gathered}
$$


where we have put

$$
x=\xi+i \eta
$$

We will divide the damain $D$ into two paris, $D_{1}$ and $D_{2}$ defined respectively by

$$
|\eta| \geqq \alpha>0 \text { and }|\eta| \leqq \alpha \text { : }
$$

$$
D=D_{i}+D_{i}
$$

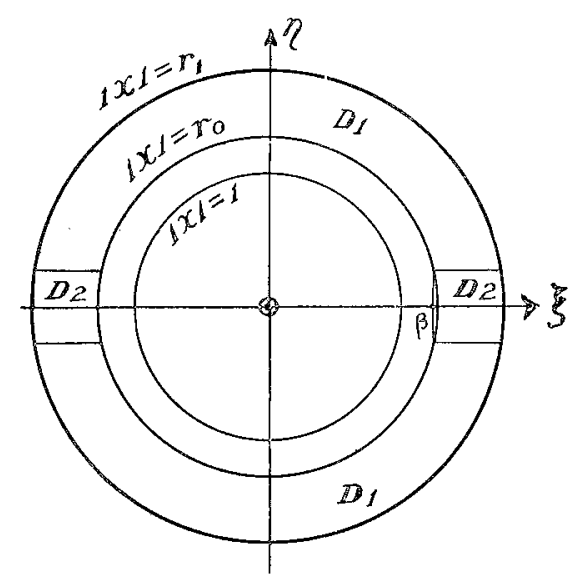

Before we begin the evaluation of the integral (6) we will prove that we can determine $n_{1}$ so that for $n \geqq n_{1}$

$$
\int_{t_{0}+\epsilon}^{1}\left(\log \frac{1}{t}\right)^{k-1} t^{n+1} d t \geqq \frac{\Gamma(k)(1-\delta)}{(n+2)^{k}}
$$

uniformly for $1<r_{0} \leqq|x| \leqq r_{1}$, where $\delta$ is a small quantity less than 1 .

To prove this, we start from the relation;

$$
\begin{gathered}
\int_{0}^{1}\left(\log \frac{1}{t}\right)^{k-1} t^{\imath+1} d t=\frac{I(k)}{(n+2)^{k}}=\int_{0}^{t_{0}+\epsilon}+\int_{t_{0}+\epsilon}^{1} \\
\int_{0}^{t_{0}+\epsilon}\left(\log \frac{1}{t}\right)^{k-1} t^{n+1} d t \leqq\left(t_{3}+\varepsilon\right)^{n+1} \int_{0}^{1}\left(\log \frac{1}{t}\right)^{k-1} d t=I^{\prime}(k)\left(t_{3}+\varepsilon\right)^{n+1}
\end{gathered}
$$

Therefore

$$
\begin{aligned}
& (n+2)^{k} \int_{0}^{t_{0}+e}\left(\log \frac{1}{t}\right)^{k-1} t^{n+1} d t \leqq \Gamma(k)(n+2)^{k}\left(t_{1}+\varepsilon\right)^{n+1} \\
& \leqq I^{\prime}(k)(n+2)^{k}\left(\tau_{0}+\varepsilon\right)^{n+1}
\end{aligned}
$$


When we choose $\varepsilon$ so small that $\tau_{0}+\varepsilon<1$; then

$$
\lim _{n=\infty}(n+2)^{n}\left(\tau_{0}+\varepsilon\right)^{n+1}=0,
$$

hence we can determine $n_{1}$ so that for $n \geqq n_{1}$

$$
(n+2)^{n}\left(\tau_{0}+\varepsilon\right)^{n+1}<\delta<1
$$

Hence

$$
\left|\int_{0}^{t_{0}+e}\left(\log \frac{1}{t}\right)^{k-1} t^{n+1} d t\right|<\frac{\delta}{(n+2)^{k}} I(k)
$$

From (8) we get for $n \equiv n_{1}$

$$
\int_{t_{0}+\varepsilon}^{i}\left(\log \frac{1}{t}\right)^{k-1} t^{n+1} d t=\frac{\Gamma(k)}{(n+2)^{k}}-\int_{0}^{t_{0}+\epsilon} \equiv \frac{\Gamma(k)(1-\delta)}{(n+2)^{k}}
$$

i.e.

$$
\int_{t_{0}+e}^{1}\left(\log \frac{1}{t}\right)^{k-1} t^{n+3} d t \geqq \frac{\Gamma^{\prime}(k)(1-\delta)}{(n+2)^{k}}
$$

which is the relation to bo proved.

Now we begin the evaluation of the integral (6).

(i) When $x$ lies in the domain $D_{1}$, we get, neglecting the first term of the radicand in (6)

$$
\begin{gathered}
\left|\int_{t_{0}+\epsilon}^{1}\left(\log \frac{1}{t}\right)^{k-1} \frac{x^{n+1} t^{2}}{x t-1} d t\right| \geq|x|^{n+1} \alpha \int_{t_{0+\epsilon}}^{1}\left(\log \frac{1}{t}\right)^{k-1} \frac{t^{n+1} d t}{|x|^{2} t+2|\xi| t+1} \\
\geqq \frac{|x|^{n+1} \alpha}{(|x|+1)^{2}} \int_{t_{0}+\epsilon}^{1}\left(\log \frac{1}{t}\right)^{k-1} t^{n+1} d t \geqq \frac{|x|^{n+1} \alpha}{\left(r_{1}+1\right)^{2}} \int_{t_{0}+\epsilon}^{1}\left(\log \frac{1}{t}\right)^{k-1} t^{n+1} d t
\end{gathered}
$$

Combining (7) and (9), we get

$$
\left|\int_{t_{0}+\mathrm{e}}^{1}\left(\log \frac{1}{t}\right)^{k-1} \frac{x^{n+1} t^{n} d t}{x t-1}\right| \geqq \frac{\left|x^{2}\right|^{n+1} \alpha \Gamma(k)(1-\delta)}{\left(r_{1}+1\right)^{2}(n+2)^{k}} \geqq \frac{|x|^{n} r_{n} \alpha \Gamma(k)(1-\delta)}{\left(r_{1}+1\right)^{2}(n+2)^{k}}
$$

Hence there exists a constant $A_{1}$, independent of $x$, such that for $n \geqq n_{1}$

$$
\left|\int_{t_{0}+e}^{1}\left(\log \frac{1}{t}\right)^{k-1} \frac{x^{n+1} t^{n}}{x t-1} d t\right| \geqq A_{1} \frac{|x|^{n}}{n^{k}}
$$

(ii) When $x$ lies in the domain $D_{2}$ and:

(a) When $\xi<0$, we have $\xi t-1<0$ and $|\xi t-1|>1$.

(b) When $\xi>0$, we will prove that by taking $\alpha$ very small, we can put 


$$
\xi t-\geqq \Sigma_{1}
$$

aniformly for $x$, when $t$ varies from $t_{0}+\approx$ to 1 ,

In fact, we can make $\beta=\sqrt{r_{0}^{2}-\alpha^{2}}>1$, since $r_{0}>1$, so that

$$
r_{1} \geqq \xi \geq \beta>1 \text {. }
$$

Hence it is sufficient to prove that

or

$$
\begin{array}{r}
\xi\left(t_{0}+\varepsilon\right)-1 \geqq \hat{s}_{1} \\
\frac{\xi}{\sqrt{\xi^{2}+\eta^{2}}}+\varepsilon \xi-1 \geqq \hat{s}_{1}
\end{array}
$$

since

$$
t_{0}=\frac{1}{|x|}=\frac{1}{\sqrt{\xi^{2}+\eta^{2}}}
$$

The inequality (12) becomes

$$
\varepsilon \xi+\frac{\xi-\sqrt{\xi^{2}+\xi^{2}}}{\sqrt{\xi^{2}+\eta^{2}}} \equiv s_{1} \quad \text { or } \quad \varepsilon \xi-\frac{\eta^{2}}{|x|(\xi+|x|)} \equiv s_{1}
$$

which is satisfied, if we take

$$
\varepsilon \beta-\frac{\alpha^{2}}{r_{0}\left(1+r_{0}\right)} \geqq \Sigma_{1}
$$

Hence we can take $\alpha$ so small, that (14) holds, if $s_{1}<\triangleq r_{0}$, since

$$
\lim _{x=0} \varepsilon \beta=r_{0} \hat{\text { s, }}
$$

For such $\alpha$ and $\varepsilon_{1}$ (11) holds evidently. Now wo see that $\xi t-1$ is positive, when $\xi$ is positive and is greater than a positive quantity $s_{1}$ and that when $\xi$ is negative, $\xi t-1$ is negative and its absolute value is greater than 1. Hence by taking $\varepsilon_{1}$ smaller than 1 , we can make $\xi t-1$ retain the same sign in the domain $D_{2}$ and have an absolute value greater than $\varepsilon_{1}$.

Now by neglecting the second term of the radicand in (6), we get

$$
\left|\int_{t_{0}+\epsilon}^{1}\left(\log \frac{1}{t}\right)^{k-1} \frac{x^{n+1} t^{n}}{x t-1} d t\right| \geqq|x|^{n+1}\left|\int_{t_{0}+\epsilon}^{1}\left(\log \frac{1}{t}\right)^{n-1} \frac{t^{n}(\xi t-1) d t}{(\xi t-1)^{2}+\eta^{2} t^{2}}\right|
$$

Since in the integral (15) $\xi t-1$ does not change sign, we do not augment the absolute value of the integral when we replace the integrand by its absolute value, we get

$$
|x|^{n+1}\left|\int_{t_{0}+\varepsilon}^{1}\left(\log \frac{1}{t}\right)^{k-1} \frac{t^{n}(\xi t-1)}{(\xi t-1)^{2}+\eta^{2} t^{2}} d t\right|=|x|^{n+1} \int_{t_{0}+\varepsilon}^{1}\left(\log \frac{1}{(k-1} \frac{t^{n}|\xi t-1|}{(\xi t-1)^{2}+\eta^{2} t^{2}} d t\right.
$$


$\geqq|x|^{n+1} \hat{s}_{1} \int_{60+\epsilon}^{1}\left(\log \frac{1}{t}\right)^{k-1} \frac{t^{n}}{\left(\xi^{2}+\eta^{2}\right) t^{2}+2|\xi| t+1} d t$

$$
\geqq|x|^{n+1} s_{1} \int_{t_{0}+\epsilon}^{1}\left(\log \frac{1}{t}\right)^{k-1} \frac{t^{n} d t}{|x|^{2} t^{2}+2|x| t+1}
$$

$\geqq \frac{|x|^{n+1} \varepsilon_{1}}{\left(r_{1}+1\right)^{2}} \int_{t_{0}+\epsilon}^{1}\left(\log \frac{1}{t}\right)^{t-1} t^{n} d t \geqq \frac{|x|^{n} r_{0} \varepsilon_{1}}{\left(r_{1}+1\right)^{2}} \int_{t_{0}+\Theta}^{1}\left(\log \frac{1}{t}\right)^{k-1} t^{n} d t$

From (7) and (16) we see that there exists a constant $A_{2}$ such that for $n \geqq n_{2}$

$$
\left|\int_{t_{0}+\epsilon}^{1}\left(\log \frac{1}{t}\right)^{k-1} \frac{x^{n+1} t^{n}}{x t-1} d t\right| \geqq \frac{A_{2}|x|^{n}}{n^{k}}
$$

By taking $A_{3}$ smaller than $A_{1}$ and $A_{2}$, we see from (10) and (17) that there exists a constant $A_{3}$, such that for $n \geqq n_{3}$

$$
\left|\int_{t_{0}+\mathrm{e}}^{1}\left(\log \frac{1}{t}\right)^{k-1} \frac{x^{n+1} t^{n}}{x t-1} d t\right| \geqq \frac{A_{3}|x|^{n}}{n^{k}}
$$

uniformly in the domain $D$.

Now from (3), (4), (5) and (18) we get,

$$
\begin{aligned}
& \left|\int_{0}^{1} \frac{1}{t}\left(\log \frac{1}{t}\right)^{k-1} \frac{x^{n+1} t^{n+1}-x t}{x t-1} d t\right| \geqq \frac{A_{3}|x|^{n}}{n^{k}}-\frac{\Gamma(k)}{\varepsilon}-n|x|\left(1+\left.\varepsilon|x|\right|^{n-1} \Gamma^{\prime}(k)\right. \\
& =\frac{A_{3}|x|^{n}}{n^{k}}\left[1-\Gamma(k)\left(\frac{1}{\varepsilon A_{3}} \frac{n^{k}}{|x|^{n}}+\frac{n}{A_{3}}\left(\frac{1+\varepsilon|x|}{|x|}\right)^{n-1}\right)\right] \\
& \geqq \frac{A_{3}|x|^{n}}{n^{k}}\left[1-\Gamma(k)\left(\frac{1}{\varepsilon A_{3}} \frac{n^{n}}{r_{0}{ }^{n}}+\frac{n}{A_{3}}\left(\frac{1+\varepsilon r_{1}}{r_{0}}\right)^{n-1}\right)\right]
\end{aligned}
$$

We will take $\varepsilon$ so small that $1+\varepsilon r_{1}<r_{0}$; this is possible since $r_{0}>1$,

Then $\lim _{n=\infty} n\left(\frac{1+\varepsilon r_{1}}{r_{0}}\right)^{n}=0$ and $\lim _{n=\infty} \frac{n^{k}}{r_{0}{ }^{n}}=0$, hence we can find $n_{4}$, independent of $x$, so that for $n \geqq n_{4}$

$$
\left|\int_{0}^{1} \frac{1}{t}\left(\log \frac{1}{t}\right)^{k-1} \frac{x^{n+1} t^{n+1}-x t}{x t-1} d t\right| \geqslant A_{3}\left(1-\delta^{\prime}\right) \frac{|x|^{n}}{n^{k}}
$$

Hence there exists a positive constant $A$, such that for $n \geqq n_{4}$

$$
\left|\frac{1}{\Gamma(k)} \int_{0}^{1} \frac{1}{t}\left(\log \frac{1}{t}\right)^{k-1} \frac{x^{n+1} t^{n+1}-x t}{x t-1} d t\right| \geqslant A \frac{|x|^{n}}{n^{k}}
$$


unifornly in the domain $D$; which proves the lemma in case $k$ is positive.

6. Proof of the lemma in case $k \leqq 0$.

When $k$ is zero or negative, we put $\alpha=-k$

Hence $\quad \alpha \geqq 0$

Consider $\quad \varphi(x)=x+2^{\alpha} x^{2}+\cdots+n^{\alpha} x^{n}=x+\frac{x^{2}}{2^{k}}+\cdots+\frac{x^{n}}{n^{k}}$

Let

And $\quad \mu=[\alpha]+1, \quad \lambda=\mu-\alpha$;

then $\quad 1 \geqq \lambda>0$.

Put $\quad \varphi_{1}(x)=x+\frac{x^{2}}{2^{\mu-\alpha}}+\cdots+\frac{x^{n}}{n^{\mu-\alpha}}=x+\frac{x^{2}}{2^{\lambda}}+\cdots+\frac{x^{n}}{n^{\lambda}}$.

then

$$
\varphi(x)=x \frac{d}{d x}\left(x\left(\frac{d}{d x}\left(\cdots x\left(\frac{d}{d x} \phi_{1}(x)\right)\right)\right)\right)
$$

$\mu$ times

Since $\lambda$ is positive, we can apply (2),

$$
\varphi_{1}(x)=\frac{1}{\Gamma(\lambda)} \int_{0}^{1} \frac{1}{t}\left(\log \frac{1}{t}\right)^{\lambda-1} \frac{x^{n+1} t^{n+1}-x t}{x t-1} d t
$$

Now we pat

$$
\begin{aligned}
& \psi_{1}(\rho)=\rho+\cdots+\rho^{n}=\frac{\rho^{n+1}-\rho}{\rho-1} \\
& \psi_{1}(\rho)=\rho \psi_{0}^{\prime}(\rho)=\rho+\cdots+n \rho^{n}=\frac{(n+1) \rho^{n+1}}{\rho-1}+\frac{\rho^{n+2}-\rho}{(\rho-1)^{2}} \\
& \psi_{2}(\rho)=\rho \psi_{1}^{\prime}(\rho)=\rho+\cdots+n^{2} \rho^{n} \\
& \cdots \cdots \cdots \cdots+\cdots \cdots+\dot{n}^{r} \rho^{n} \\
& \psi_{r}(\rho)=\rho \psi_{r-1}^{\prime}(\rho)=\rho+\cdots \cdots
\end{aligned}
$$

We can prove by induction that

$$
\psi_{r}(\rho)=\frac{(n+1)^{r} \rho^{n+1}}{\rho-1}+\frac{\theta_{r}}{(\rho-1)^{r+1}},
$$

$\theta_{r}$ being a polynomial of the $(n+r+1)$-th degree at most:

where

$$
\theta_{\tau}=a_{0}+a_{1} \rho+\cdots+a_{n+r+1} \rho^{n+r+1}
$$

$$
\left|a_{i}\right|<K(n+1)^{r-1}
$$


$K$ being a function of $r$, but independent of $n$.

Hence

$$
\begin{aligned}
& \left|\frac{\theta_{r}}{(n+1)^{r} \rho^{n+1}}\right| \leqq \frac{K}{n+1} \frac{\left(1+|\rho|+\cdots+|\rho|^{n+r+1}\right)}{|\rho|^{n+1}}=\frac{K}{n+1} \frac{|\rho|^{n+r+2}-1}{(|\rho|-1) \mid \rho^{n+1}} \\
& \quad \leqq \frac{K}{n+1} \cdot \frac{|\rho|^{r+1}}{|\rho|-1} \leqq \frac{K \rho_{1}^{r+1}}{(n+1)\left(\rho_{0}-1\right)},
\end{aligned}
$$

for $1<\rho_{0} \leqq \rho \mid \leqq \rho_{1}$.

Therefore

$$
\lim _{n \rightarrow \infty} \frac{\theta_{r}}{(n+1)^{r} \rho^{n+1}}=0
$$

uni̊rormly for $1<\rho_{0} \leqq|\rho| \leqq \rho_{1}$.

Now

$$
x \frac{d}{d x}\left(\frac{x^{n+1} t^{n+1}-x t}{x t-1}\right)=x t \frac{d}{d(x t)}\left(\frac{x^{n+1} t^{n+1}-x t}{x t-1}\right)=\rho \frac{d}{d \rho}\left(\frac{\rho^{n+1}-\rho}{\rho-1}\right)=\psi_{1}(\rho),
$$

where

$$
\rho=x t \text {. }
$$

From (20) (21) (25) we get,

$$
\varphi(x)=\frac{1}{\Gamma(1)} \int_{0}^{1} \frac{1}{t}\left(\log \frac{1}{t}\right)^{\lambda-1} \psi_{\mu}(x t) d t
$$

In order to evaluate the integral (25), we divide it into two parts:

$$
\int_{0}^{1}=\int_{0}^{t_{0}+\epsilon}+\int_{t_{j}+\epsilon}^{1}
$$

where

$$
t_{0}=\frac{1}{|x|} \text {. }
$$

a) $\left|\int_{0}^{0+e} \frac{1}{t}\left(\log \frac{1}{t}\right)^{\lambda-1} \psi_{\mu}(x t) d t\right| \leqq \int_{0}^{t_{0}+e}\left(\log \frac{1}{t}\right)^{\lambda-1}\left(\left.\left|x_{1}+\cdots+n^{\mu}\right| x\right|^{n} t^{n-1}\right) d t$

$$
\begin{aligned}
& <n^{\mu+1}\left|x_{1}^{n}\left(t_{0}+\varepsilon\right)^{n-1} \int_{0}^{t_{0}+\epsilon}\left(\log \frac{1-}{t}\right)^{\lambda-1} d t<n^{\mu+1}\left(1+z_{i}^{\prime} x \mid\right)^{n-1}\right| x||_{0}^{1}\left(\log \frac{1}{t}\right)^{\lambda-1} d t \\
& \leqq n^{\mu+1} r_{1}\left(\mathbf{1}+\varepsilon \gamma_{i}\right)^{n-1} \Gamma(\lambda)
\end{aligned}
$$

Hence there exists a constant $A_{4}$, such that 


$$
\left|\int_{0}^{t_{0}+e} \frac{1}{t}\left(\log \frac{1}{t}\right)^{\lambda-1} \psi_{\mu}(x t) d t\right|<A_{4} n^{\mu+1}\left(1+\varepsilon r_{1}\right)^{n}
$$

b) $\left|\int_{t_{0}+\varepsilon}^{1} \frac{1}{t}\left(\log \frac{1}{t}\right)^{\lambda-1} \psi_{\mu}(x t) d t\right| \geqq\left|\int_{t_{0}+\varepsilon}^{1} \frac{1}{t}\left(\log \frac{1}{t}\right)^{\lambda-1} \frac{(n+1)^{\mu} x^{n+1} t^{n+1}}{x t-1} d t\right|$

$$
-\left|\int_{t 0+\epsilon}^{1}-\frac{1}{t}\left(\log \frac{1}{t}\right)^{\lambda-1} \frac{\theta_{\mu}}{(x t-1)^{\mu+1}} d t\right|
$$

c) When $t$ varies from $t_{0}+\varepsilon$ to 1 and $x$ lies in the domain $D$,

$$
|x|\left(t_{0}+\varepsilon\right) \leqq|x t| \leqq r_{1}
$$

Since

$$
|x|\left(t_{n}+s\right)=1+s|x| \geq 1+s r_{3}>1
$$

we get

$$
1<1+\varepsilon r_{0} \leqq|x t| \leqq r_{1}
$$

Hence from (24) we can determine $n_{5}$ so that for $n \geq n_{5}$

$$
\left|\frac{\theta_{i n}}{(n+1)^{\mu}\left(x_{t}\right)^{n+1}}\right| \leqq s_{1}
$$

uniformly in the domain $D$, when $t$ varies from $t_{3}+\varepsilon$ to $I$.

Now $\int_{t_{0}+\varepsilon}^{1} \frac{1}{t}\left(\log \frac{1}{t}\right)^{\lambda-1} \frac{\theta_{\mu}}{(x t-1)^{\mu+1}} d t$

$\leqq s_{1} \int_{t_{0}+\epsilon}^{1} \frac{1}{t}\left(\log \frac{1}{t}\right)^{\lambda-1} \frac{(n+1)^{\mu} \mid x t !^{n+1}}{(|x t|-1)^{n+1}} d t$

$\leqq \frac{\varepsilon_{1}(n+1)^{\mu}|x|^{n+1}}{\varepsilon^{\mu+1} r_{3}^{\mu+1}} \int_{t_{0}+\epsilon}^{1}\left(\log \frac{1}{t}\right)^{\lambda-1} t^{n} d t<\frac{\varepsilon_{1}(n+1)^{\mu}|x|^{n+1}}{\varepsilon^{\mu+1} v_{0}^{\mu+1}} \int_{0}^{1}\left(\log \frac{1}{t}\right)^{\lambda-1} t^{n} d t$

$=\frac{\varepsilon_{1}(n+1)^{\mu}|x|^{n+1}}{\varepsilon^{\mu+1} r_{0}^{\mu-1}} \frac{\Gamma(\lambda)}{(n+1)^{\lambda}}=\frac{\varepsilon_{1}(n+1)^{\alpha}|x|^{i n+1} \Gamma(i)}{s^{\mu+1} r_{j}^{\mu+1}} \leqq B n^{\alpha}|x|^{n} \xi_{1}$,

where $B$ is a constant.

Hence

$$
\left|\int_{t 0+\varepsilon}^{1} \frac{1}{t}\left(\log \frac{1}{t}\right)^{\lambda-1} \frac{\theta_{\mu}}{(x t-1)^{\mu-1}} d t\right|<B n^{\alpha}|x|^{n}=_{1}
$$


d) $\quad \mid \int_{0^{+}+\epsilon}^{1} \frac{1}{t}\left(\log \frac{1}{t}\right)^{\lambda-1} \frac{x^{n+1} t^{n+1}(n+1)^{\mu}}{x t-1} d t$

$$
=|x|^{n+1}(n+1)^{\mu}\left|\int_{t_{0}+e}^{1}\left(\log \frac{1}{t}\right)^{\lambda-1} \frac{t^{n} d t}{x t-1}\right|
$$

By (17) there exists a constant $A_{3}$, such that for $n \geqq n_{3}$.

$$
|x|^{n+1}(n+1)^{\alpha}\left|\int_{t_{0}+e}^{1}\left(\log \frac{1}{t}\right)^{\lambda-1} \frac{t^{n} d t}{x t-1}\right| \geqq|x|^{n} \frac{A_{3} n^{\mu}}{n^{\lambda}}
$$

Therefore

$$
\left|\int_{t_{0}+\epsilon}^{1} \frac{1}{t}\left(\log \frac{1}{t}\right)^{\lambda-1} \frac{x^{n+1} t^{n+1}(n+1)^{\mu}}{x t-1} d t\right| \geqq A_{3}|x|^{n} n^{\alpha}
$$

e) From (27) (28) (31) (33) we get

$$
\begin{aligned}
& \left|\int_{0}^{1} \frac{1}{t}\left(\log \frac{1}{t}\right)^{\lambda-1} \Psi_{n}(x t) d t\right| \geqq A_{3}|x|^{n} n^{\alpha}-B n^{\alpha}|x|^{n} \hat{s}_{1}-A_{1} n^{\mu+1}\left(1+\varepsilon r_{1}\right)^{\mu} \\
& \geqq A_{3}|x|^{n} n^{\alpha}\left(1-\frac{B}{A_{3}} \hat{\varepsilon}_{1}-\frac{A_{4}}{A_{3}} n^{\lambda+}\left(\frac{1+\varepsilon r_{1}}{r_{0}}\right)^{n}\right) .
\end{aligned}
$$

Now we choose $\varepsilon$ and $\varepsilon_{1}$ so that $4 \frac{B}{A_{3}} \varepsilon_{1} \leqq 1 ; 1+\varepsilon r_{1}<r_{u}$, then $\lim _{n \rightarrow \infty} n^{\lambda+1}\left(\frac{1+\varepsilon r_{1}}{r_{0}}\right)^{n}=0$, hence we can find $n_{\mathfrak{6}}$, such that for $n \geqq n_{e}$

$$
\left|\frac{A_{4}}{A_{3}} n^{\lambda+1}\left(\frac{1+\varepsilon r_{1}}{r_{0}}\right)^{n}\right| \leqq \frac{1}{4}
$$

Therefore

$$
\left|\int_{0}^{1} \frac{1}{t}\left(\log \frac{1}{t}\right)^{\lambda-1} \psi_{n}(x t) d t\right| \geqq A_{3}|x|^{n} n^{\alpha}\left(1-\frac{1}{4}-\frac{1}{4}\right)=\frac{A_{3}}{2}|x|^{n} n^{\alpha} .
$$

Hence there exists a constiant $A$ so that for $n \geqq n_{7}$

$$
|\varphi(x)|=\left|\frac{1}{\Gamma(\lambda)} \int_{0}^{1} \frac{1}{t}\left(\log \frac{1}{t}\right)^{\lambda-1} \psi_{n}(x t) d t\right| \geqq A|x|^{n} n^{a}=\frac{A|x|^{n}}{n^{i}}
$$

uniformly in the domain $D$, which proves the lemma.

7. Lemma II. In the domain $D$ defined by $1<r_{0} \leqq|x| \leqq r_{1}$ there exisets a constant $B$, such that for $n \geqq n_{0}$

$$
1+\frac{|x|}{1}+\cdots+\frac{|x|^{n}}{n^{k}} \leq B \frac{|x|^{n}}{n^{k}}
$$


where $k$ is any real number.

Proof. We put

and

$$
|x|=r^{*}
$$

$$
f(t)=\frac{r^{*}}{t^{k}}
$$

then

$$
f^{\prime}(t)=\frac{r^{t}}{t^{k}}\left(\log r-\frac{k}{t}\right)
$$

Hence $f^{\prime}(t)>0$ for $\log r>\frac{|k|}{t}$ or $t>\frac{|k|}{\log r}$.

Therefore in the domain $D$

$$
f^{\prime}(t)>0
$$

if we take

$$
t>\frac{|k|}{\log r_{0}}
$$

Similarly putting

$$
\psi(t)=\frac{r^{t}}{t^{k+1}}
$$

we see that

$$
\psi^{\prime}(t)>0
$$

uniformly in the domain $D$, if we take

$$
t>\frac{|k|+1}{\log r_{0}}
$$

Hence when we denote by $n_{0}$ any natural number greater than $\frac{|k|+1}{\log r_{0}}, f(t)$ and $\psi(t)$ are monotonously increasing for $t \geq n_{0}$, uniformly in the domain $D$. Now we put

$$
B_{1}=1+\frac{r_{1}}{1}+\cdots+\frac{r_{1}^{n_{0}-1}}{\left(n_{0}-1\right)^{k}}
$$

T'hen, since $f(t)$ is increasing, we get,

$$
1+\frac{|x|}{1}+\cdots+\frac{|x|^{n}}{n^{k}} \leqq B_{1}+\frac{|x|^{n_{0}}}{n_{0}^{k}}+\cdots+\frac{|x|^{n}}{n^{k}}<B_{1}+\int_{n_{0}}^{n} \frac{|x|^{t}}{t^{k}} d t+\frac{|x|^{n}}{n^{k}}
$$




$$
\leq B_{1}+\left[\frac{|x|^{t}}{\log |x| t^{k}}\right]_{n_{0}}^{n}+\frac{|k|}{\log |x|} \int_{n b_{0}}^{n} \frac{|x|^{t}}{t^{k+1}} d t+\frac{|x|^{n}}{n^{k}}
$$

And since $\frac{|x|^{t}}{t^{k+1}}$ is also increasing, we gat

$$
\int_{n_{0}}^{n} \frac{|x|^{t}}{t^{k+1}} d t<n \frac{x^{n}}{n^{n+1}}=\frac{x^{n}}{n^{k}}
$$

Hence

$$
\begin{aligned}
1+\frac{|x|}{1}+\cdots & +\frac{|x|^{n}}{n^{k}}<B_{1}+\frac{|x|^{n}}{\log |x| n^{k}}+\frac{|k|}{\log |x|} \frac{|x|^{n}}{n^{k}}+\frac{|x|^{n}}{n^{k}} \\
& \leqq \frac{x n^{n}}{n^{k}}\left(1+\frac{|k|+1}{\log r_{0}}+B_{1} \frac{n^{k}}{r_{\nu}{ }^{n}}\right) .
\end{aligned}
$$

Since $\lim _{n=\infty} \frac{n^{k}}{r_{0}{ }^{n}}=0$, there exists a constant $B$, such that for $n \geqq n_{0}$

$$
1+\frac{|x|}{1}+\cdots+\frac{|x|^{n}}{n^{k}} \leqq \mathrm{~B} \frac{|x|^{n}}{n^{k}}
$$

which proves the lemma.

8. Lemma III. In the domain $D$ defined by $1<r_{0} \leqq|x| \leqq r_{1}$ there exists a positive constant $M I$, such that for $n \equiv n_{0}$

$$
\left|1+\frac{x}{1}+\frac{x^{2}}{n^{k}}+\cdots+\frac{x^{n}}{n^{k}}\right| \geqq M\left(1+\frac{|x|}{1}+\cdots+\frac{|x|^{n}}{n^{k}}\right)
$$

where $k$ is any real number.

Proof. We have

$$
\left|1+\frac{x}{1}+\cdots+\frac{x^{n}}{n^{n}}\right| \geqq\left|\frac{x}{1}+\cdots+\frac{x}{n}\right|-1
$$

By Lemma $\mathrm{I}$

$$
\left|\frac{x}{1}+\cdots+\frac{x^{n}}{n^{k}}\right| \geqq A\left|\frac{x^{n}}{n^{k}}\right|,
$$

for $n \geq n_{0}$

Hence there exists a constant $C$ such that for $n \geqq n_{0}$

$$
\left|1+\frac{x}{1}+\cdots+\frac{x^{n}}{n^{k}}\right| \geqq A \frac{|x|^{n}}{n^{k}}-1 \geq A \frac{|x|^{n}}{n^{k}}\left(1-\frac{n^{k}}{r_{0}{ }^{n}}\right)>C \frac{|x|^{n}}{n^{k}},
$$

uniformly in the domain $D$. 
By Lemma II and (38) we get

$$
\left|1+\frac{x}{1}+\cdots+\frac{x^{n}}{n^{k}}\right| \geqq \frac{C}{B}\left(1+\frac{|x|}{1}+\cdots+\frac{|x|^{n}}{n^{k}}\right)=M\left(1+\frac{|x|}{1}+\cdots+\frac{x^{n}}{n^{k}}\right)
$$

for $n \geq n_{0}$, uniformly in the domain $D, \pi$ bing a positive constant.

3.

9. Now we begin to prove our fundamental theorem II :

Theorem II. If in a pcuer series

$$
F(\boldsymbol{x})=a_{0}+a_{1} x+a_{2} x^{2}+\cdots+a_{n} x^{n}+\cdots
$$

$\lim _{n \rightarrow \infty} \frac{a_{i n}}{n^{k}}=A(\neq 0)$, where $k$ is any real number, then there are no limiting points of the wero points of sections outside the unit-circle.

Proof.

Since $\lim _{n=\infty} \frac{a_{n}}{n^{k}}=A$, we can put

$$
a_{n 2}=A n^{k}+s_{i n} n^{k} ; \lim _{n=s} s_{3 k}=0
$$

Suppose that the zero points of sections

$$
a_{\mathrm{u}}+a_{1} x+\cdots+a_{n} x^{n}
$$

have a limiting point $z$ outside the unit circle, which is now the convergence circle of $F^{\prime}(x)$, then we can choose a set $n_{n} n_{1} n_{2} \ldots$ such that $x_{0}, x_{1}, x_{2}, \ldots$ where $x_{i}$ is a root of the $n_{i}$-th section, converge to $z_{0}$. We now take two circles, whose radii are $r_{0}=\left|z_{0}\right|-\varepsilon$, and $r_{1}=\left|z_{0}\right|+\varepsilon$, and whose centres are at the origin. When $\varepsilon$ is very small, the two circles lie outside the unit circle. We may suppose that all the points $x_{0} x_{1} \cdots$ lie within the ring bounded by the two circles, so that

$$
r_{0} \leqq\left|x_{i}\right| \leqq r_{1} \quad(i=0,1,2 \cdots)
$$

By Lemma III, there exists a constant $M$, such that for $n \geqq n_{0}$

$$
\left|1+1^{k} x_{i}+2^{k} x_{i}{ }^{2}+\cdots+n_{i}{ }^{k} x_{i}{ }^{n_{i}}\right| \geqq M\left(1+1^{k}\left|x_{i}\right|+\cdots+n_{i}^{k}\left|x_{i}{ }^{n_{i}}\right|\right)
$$

\section{Hence}

$$
\left|1+1^{k} x_{i}+2^{k} x_{i}{ }^{2}+\cdots n_{i}^{k} x_{i}^{n^{i}}\right| \neq 0 .
$$

Now by the hypothesis

$$
a_{0}+a_{1} x_{i}+\cdots+a_{n !} x_{n_{i}}^{n_{i}}=0 . \quad(i=0,1,2, \cdots)
$$


or from (39)

$$
A\left(1+1^{k^{k} x_{i}}+\cdots+n_{i}{ }^{k} x_{i}^{n_{i}}\right)+\left(s_{0}+s_{1} x_{i}+\cdots+s_{n_{i}} n_{i}{ }^{k} x_{i}^{n_{i}}\right)=0
$$

By putting

$$
\begin{aligned}
& b_{i o}=\frac{1}{1+1^{b} x_{i}+\cdots+n_{i}{ }^{k} x_{i}{ }^{n_{i}}} \\
& b_{i r}=\frac{r^{k} x_{i}{ }^{r}}{1+1^{k} x_{i}+\cdots+n_{i}{ }^{k} x_{i}{ }^{n_{i}}} \quad(r=1,2, \cdots)
\end{aligned}
$$

Equations (42) can be put in the following form in virtue of (40).

$$
A+b_{i o} \hat{s}_{0}+b_{i 1} \varepsilon_{1}+\cdots+b_{i n_{i}} \varepsilon_{n_{l}}=0
$$

Consider the sequence $\left\{x_{i}{ }^{1}\right\}$ :

$$
\begin{gathered}
x_{i}{ }^{1}=b_{i 0} \varepsilon_{0}+b_{l 1} \varepsilon_{1}+\cdots+b_{i} n_{i} \varepsilon_{n i} \\
(i=0,1,2, \cdots)
\end{gathered}
$$

We have,

(i) $\left|b_{i 0}\right|+\left|b_{i 1}\right|+\cdots+\left|b_{i} n_{i}\right|=\frac{1+1^{k}\left|x_{i}\right|+\cdots+n_{i}^{k}\left|x_{i}\right| n_{i}}{\left|1+1^{k} x_{i}+\cdots+n_{i}^{k} x_{i} n_{i}\right|} \leqq \frac{1}{M}$.

(ii) $\left|b_{i r}\right|=\frac{r^{k}\left|x_{i}\right|^{r}}{\left|1+1^{k} x_{i}+\cdots+n_{i}^{k} x_{i}{ }^{n_{i}}\right|}<\frac{1}{M I} \frac{r^{k}\left|x_{i}\right|^{r}}{1+1^{k}\left|x_{i}\right|+\cdots+n_{i}^{k}\left|x_{i}\right| n_{i}}$

$$
\begin{aligned}
& <\frac{1}{M} \frac{r^{k}}{n_{i}^{k}\left|x_{i}\right|^{n_{i}-r}} \leqq \frac{1}{M} \frac{r^{k^{k}}}{n_{i}^{k} r_{0}^{n_{i}-r}}, \\
& \left|b_{i o}\right| \leqq \frac{1}{M} \frac{1}{1+{ }^{k}\left|x_{i}\right|+\cdots+n_{i}{ }^{k}\left|x_{i}\right|^{n_{i}}}<\frac{1}{M} \frac{1}{n_{i}{ }^{k}\left|x_{i}\right| n_{i}} \leqq \frac{1}{M}-\frac{1}{n_{i}{ }^{k} r_{o}{ }^{n_{i}}}
\end{aligned}
$$

Hence

$$
\lim _{i=\infty}\left|b_{i 0}\right|=0, \quad \lim _{i=\infty}\left|b_{i 0} r\right|=0 \quad \text { (for a fixed } r \text { ) }
$$

Now by Toepliti's Theorem $\left({ }^{1}\right)$ the sequence $\left\{x_{i}{ }^{1}\right\}$ tends to zero, when $\varepsilon_{i}$ tends to zero. But from (43), this contradicts the hypothesis that $A$ is not zero. Hence there is no limiting point outside the unit circle, which proves the theorem.

(1) Toeplitz: Über lineare Mittelbildungen, Prace matematyczno-fizyczne.

22. or Knopp. Theorie und Anwendung dex unendlichen Reihen, 2. Aufl. 392. 
Remark (i) The above result is not true, when we only suppose that $\lim _{n=\infty} \frac{\left|a_{n}\right|}{n^{k}}=A(\neq 0)$, as the following example shows. In the power series

$$
F(x)=\left(1-x-x^{2}\right)+\left(x^{3}-x^{4}-x^{5}\right)+\left(x^{3}-x^{7}-x^{8}\right)+\ldots \ldots ;\left|a_{n}\right|=1
$$

the sections;

$$
1-x-x^{2}, \quad 1-x-x^{2}+\left(x^{3}-x^{4}-x^{5}\right), \text { ete. }
$$

have the roots of $1-x-x^{2}=0$ in common, one of which $x_{1}=\frac{-1-\sqrt{5}}{2}$ has an absolute value greater than 1 .

Remark (ii) When the coefficients $a_{n}$ satisfy the condition of Theorem II, the radius of convergence is 1 and from Theorem I, the zero points of sections are bounded.

\section{4}

10. We will give some examples, where the condition of Theorem II is satisfied.

(1) $\tan ^{-1} x=x-\frac{x^{3}}{3}+\frac{x^{5}}{5}-\ldots+(-1)^{n} \frac{x^{2 n+1}}{2 n+1}+\ldots$

or

$$
\frac{1}{i \sqrt{z}} \tan ^{-1}(i \sqrt{z})=1+\frac{z}{3}+\ldots+\frac{2^{n}}{2 n+1}+\ldots .
$$

Here $a_{n}=\frac{1}{2 n+1}, \quad \quad \lim _{n=\infty} a_{n} n=\frac{1}{2}$.

(2) $\sin ^{-1} x=x+\frac{1}{2} \frac{x^{3}}{3}+\frac{1.3}{2.4} \frac{x^{5}}{5}+\ldots+\frac{1.3 . .(2 n-1)}{2.4 \ldots 2 n} \frac{x^{2 n+1}}{2 n+1}+\ldots$

or

$$
\begin{gathered}
\frac{\sin ^{-1} \sqrt{z}}{\sqrt{z}}=1+\frac{1}{2} \frac{z}{3}+\ldots \ldots+a_{n} z^{n}+\ldots \ldots \\
\lim _{n=\infty} a_{n} n^{\frac{1}{2}}=\frac{1}{2 \sqrt{\pi}} .
\end{gathered}
$$

(3) $\log \frac{1}{1-x}=x+\frac{x^{2}}{2}+\ldots+\frac{x^{n}}{n}+\ldots \ldots$

Here $\quad \lim _{n=\infty} a_{n} n=1$.

(4) The binomial series

$$
(1-x)^{r}=\Sigma a_{n} x^{n}
$$


where $r$ is any real number which is not a positive integer.

Then $\quad a_{n}=\frac{-r\left(1-\frac{r}{1}\right) \ldots\left(1-\frac{r}{n-1}\right)}{n}=-\frac{r\left(1-\frac{r}{1}\right) \ldots\left(1-\frac{r}{n}\right)}{n-r}$

Take $m$ so large that for $n>m, \quad\left|\frac{r}{n}\right|<1$,

then

$$
a_{n}=\frac{A p_{n}}{n-r}
$$

where

$$
A=-r\left(1-\frac{r}{1}\right) \ldots\left(1-\frac{r}{m}\right) ; \quad p_{n}=\left(1-\frac{r}{m+1}\right) \ldots\left(1-\frac{r}{n}\right) .
$$

Now, if in the product $p_{n}=\left(1+\alpha_{1}\right)\left(1+\alpha_{n 2}\right) \ldots\left(1+\alpha_{n}\right),\left|\alpha_{k}\right|<1$ and $\sum_{k=1}^{\infty}\left|\alpha_{k}\right|^{2}$ converges, then $\left({ }^{2}\right)$

$$
\lim _{n=\infty} \frac{p_{n}}{e^{x} n}=B(\neq 0)
$$

where

$$
s_{n}=\alpha_{1}+\ldots+\alpha_{n}
$$

Hence

$$
\begin{aligned}
& p_{n}=\left(B+\varepsilon_{n}\right) e^{s_{2 n}} ; \quad \quad \lim _{n=\infty} \varepsilon_{n}=0 \\
& a_{n}=\frac{A}{n-r}\left(B+\varepsilon_{n}\right) e^{s_{n}}=\frac{A}{n-r}\left(B+\varepsilon_{n}\right) e^{-\left(\frac{1}{m+1}+\ldots+\frac{1}{n}\right) r}= \\
& \frac{A}{n-r}\left(B+\hat{\varepsilon}_{n}\right) e^{-\left(\frac{1}{1}+\ldots+\frac{1}{n}\right) r+\left(1+\frac{1}{2}+\ldots+\frac{1}{m}\right) r}= \\
& \frac{A}{n-r}\left(B+\varepsilon_{n}\right) e^{-\left(\log n+\gamma_{n} i r+\left(1+\frac{1}{2}+\cdots+\frac{1}{m}\right)^{r}\right.}=\frac{A\left(B+\hat{\varepsilon}_{n}\right)}{(n-r) n^{r}} e^{r\left(1+\ldots+\frac{1}{m}-\gamma_{n}\right)},
\end{aligned}
$$

where

$$
\lim _{n=\infty} \gamma_{n}=\gamma \text { (Euler's constant). }
$$

$\left({ }^{2}\right)$ Knopp, Theorie und Anwendang der unondlichon Reihen, 2. Aufl. 226. 
Consequently

$$
\lim _{n=\infty} a_{n} n^{\gamma+1}=A B e^{r\left(1+\ldots+\frac{1}{m}-\gamma\right)} \neq 0
$$

Specially, when $r$ is negative, $r=-\lambda(\lambda>0)$, we know from the theory of $\Gamma$-function that

$$
\lim _{n=\infty} \frac{B_{n}^{(\lambda)}}{n^{\lambda-1}}=\frac{1}{\Gamma(\lambda)}
$$

where we have put

$$
\frac{1}{(1-x)^{\lambda}}=\sum_{n=0}^{\infty} E_{n}^{(\lambda)} x^{n}
$$

This case admits also simple verification. In fact

$$
B_{n}^{(\lambda)}=\frac{\lambda(\lambda+1) \ldots(\lambda+n-1)}{n !},
$$

so that

$$
\begin{array}{ll}
B_{n+1}^{(\lambda)}>B_{n}^{(\lambda)}, & \text { if } \lambda>1 ; \\
B_{n+1}^{(\lambda)}<B_{n}^{(\lambda)}, & \text { if } \lambda<1 .
\end{array}
$$

Hence by Kakeya's theorem we see that if $\lambda<1$, all roots of sections lie outside the unit circle, while if $\lambda<1$, they all lie inside it, however in each case as we have seen above, they converge to the unit circle; the case $\lambda=1$ is tivial; all roots lie on the unit circle.

(5) The hypergeometric series.

$$
F(\alpha . \beta, \gamma ; x)=1+\frac{\alpha, \beta}{1 . \gamma} x+\frac{\alpha(\alpha+1) \beta(\beta+1)}{2 ! \gamma(\gamma+1)} x^{2}+\ldots
$$

where $\alpha, \beta, \gamma$, are real numbers.

Here $\quad a_{n}=\frac{\alpha(\alpha+1) \ldots(\alpha+n-1) \beta(\beta+1) \ldots(\beta+n-1)}{n ! \gamma(\gamma+1) \ldots(\gamma+n-1)}$.

Now as have shewn under (4) we have

$$
\begin{array}{ll}
\frac{1}{(1-x)^{\alpha}}=\sum_{0}^{\infty} \frac{\alpha(\alpha+1) \ldots(\alpha+n-1)}{n !} x^{n}=\sum_{0}^{\infty} b_{n} x^{n} \quad ; \lim _{n=\infty} b_{n} n^{-\alpha+1}=A_{1}(\neq 0) \\
\frac{1}{(1-x)^{\beta}}=\sum_{0}^{\infty} \frac{\beta(\beta+1) \ldots(\beta+n-1)}{n !} x^{n}=\sum_{0}^{\infty} c_{n} x^{n} \quad ; \lim _{n=\infty} c_{n} n^{-\beta+1}=A_{2}(\neq 0)
\end{array}
$$




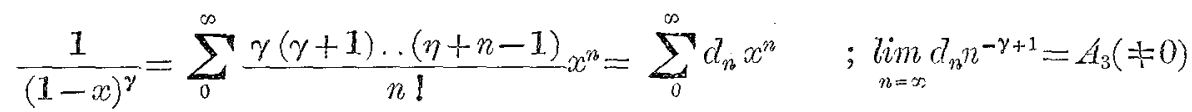

Consequently

$$
\lim _{n=\infty} a_{n n^{2} n^{1+\gamma-\alpha-\beta}}=\frac{A_{1} A_{2}}{A_{3}} \neq 0 \quad \text { since } \quad a_{n}=\frac{b_{n} c_{n}}{d_{n}} .
$$

(6) The rational function

$$
R(x)=\frac{P(x)}{Q(x)}=\frac{\alpha_{0}+\alpha_{1} x+\ldots+\alpha_{m} x^{n}}{\beta_{0}+\beta_{1} x+\ldots+\beta_{n} x^{i n}}=a_{0}+\alpha_{1} x+\ldots+a_{n} x^{n}+\ldots
$$

where we suppcse $Q(x)$ to have been brought, by a suitable linear transformation, in the following form:

$$
\begin{aligned}
Q(x) & =(1-x)^{n_{0}}\left(x_{1}-x\right)^{n_{1}}\left(x_{2}-x\right)^{n_{2}} \ldots \\
1 & \leqq \\
& \left|x_{1}\right| \leqq\left|x_{2}\right| \leqq \ldots
\end{aligned}
$$

Decomposing into partial frractions, let

$$
\begin{aligned}
R(x)= & \frac{b_{00}}{(1-x)^{n_{0}}}+\frac{b_{01}}{(1-x)^{n_{0}-1}}+\ldots+\frac{b_{0, n_{0}-1}}{1-x}+ \\
& \frac{b_{10}}{\left(1-\frac{x}{x_{1}}\right)^{n_{1}}}+\frac{b_{11}}{\left(1-\frac{x}{x_{1}}\right)^{n_{1}-1}}+\ldots+\frac{b_{1, n_{1}-1}}{1-\frac{x}{x_{1}}}+\ldots \ldots \ldots \ldots \\
& +c_{0}+c_{1} x+\ldots+c_{r} x^{r} .
\end{aligned}
$$

Then for $n>r$,

$$
\begin{aligned}
a_{n}= & b_{00} B_{n}^{\left(n_{0}\right)}+b_{01} B_{n}^{\left(n_{0}-1\right)}+\ldots+b_{0, n_{0-1}} \\
& +\left(b_{10} B_{n}^{\left(n_{1}\right)}+b_{11} B_{n}^{\left(n_{1}-1\right)}+\ldots+b_{1, n_{1}-1}\right)\left(\frac{1}{x_{1}}\right)^{n}+\ldots
\end{aligned}
$$

and, as we can easily verify,

$$
\lim _{n=\infty} \frac{B_{n}^{\left(n_{1}-i\right)}}{B_{n}^{(n o)}}=0, \quad \lim _{n \rightarrow \infty} \frac{B_{n}^{\left(n_{1}\right)}}{B_{n}^{\left(n_{0}\right)}}\left(\frac{1}{x_{1}}\right)^{n}=0, \quad \text { if } \quad\left|x_{1}\right|>1
$$

Suppose now $n_{0}=n_{1}==n_{k}$ and $1=\left|x_{1}\right|=\left|x_{2}\right|=\left|x_{k}\right|$; and as to the other roots, let $\left|x_{k+i}\right|>1$ or $n_{k+i}<n_{0}$ if $\left|x_{k+i}\right|=1$, then we can put, in virtue of $(58)$

$$
\frac{a_{n}}{n^{n-1}-1}=\left(b_{00}+\frac{b_{10}}{x_{1}^{n_{0}}}+\ldots+\frac{b_{k 0}}{x_{k}{ }^{n}}+\varepsilon_{n n}\right) \frac{1}{\Gamma\left(n_{0}\right)}
$$


where

since

$$
\begin{gathered}
\lim _{n=\infty} \varepsilon_{n}=0, \\
\lim _{n=\infty} \frac{B_{n b}^{(\lambda)}}{n^{\lambda-1}}=\frac{1}{I^{\prime}(\lambda)}
\end{gathered}
$$

Hence iI $\lim _{n=\infty}\left(b_{00}+\frac{b_{3 n}}{x_{1}^{n}}+\ldots+\frac{b_{k i n}}{x_{k}^{n}}\right) \neq 0$, we have $\lim _{n=\infty} \frac{a_{n}}{n^{n_{0}-1}} \neq 0$.

When the condition in the following theorern is satisfied, the above limit certainly exists, hence we get

Theorem III. If in a rational function $R(x)=\frac{P(x)}{Q(x)}=a_{0}+a_{1} x+\ldots$

$$
Q(x)=\left(x-x_{0}\right)^{n_{0}}\left(x-x_{1}\right)^{n_{1}} \ldots\left(x-x_{\nu}\right)^{n_{\nu}},
$$

and

i) $\left|x_{0}\right|=\left|x_{1}\right|=\ldots=\left|x_{k}\right|<\left|x_{k+1}\right| \leqq \ldots \leqq\left|x_{\nu}\right|$

ii) $n_{0}, n_{1} \ldots n_{k}$ are all different, then there are no limiting points of the zero points of sections of the expansion of $R(x)$ outsicle the circle $|x|=|x$,$| .$

5.

11. In the following we will investigate the distribution of the zero points of sections of a transcendental integral function.

It has been proved by Prof. Okada( $\left(^{3}\right)$ that a necessary and sufficient condition that

$$
F^{\prime}(x)=a_{0}+a_{1} x+\ldots+a_{n} x^{n}+\ldots
$$

is a transcendental integral function is that

$$
\lim _{n=\infty} r_{n b}=\infty
$$

where $r_{n}$ is the maximum modulus of the zero points of the $n$-th section.

The original proof of the theorem is very complicated. The following simple proof is due to Prof. Kakeya, who has authorized us to insert it here.

Theorem IV. (Kakeya)

(3) The science reports of the Toholku Imperial University, 11 (1922), 43-50. 
Let a power series $\sum_{n=0}^{\infty} a_{n} x^{n}$ have a convergence-radius $r$, then by taking suitable sections :

$$
S_{m_{i}}(x)=\sum_{n=0}^{m_{i}} a_{n} x^{n}, \quad(i=1,2,3 \ldots)
$$

we can make $\lim _{i=\infty} S_{m i}(x)=\infty$ uniformly for $|x|>k>2 r$.

Proof. Since $\frac{k}{2}>r$, we get

$$
\varlimsup_{n=\infty}\left|a_{n}\right|\left(\frac{k}{2}\right)^{n}=\infty
$$

Hence if we take $m_{i}$ suitably,

$$
\left|a_{m}\left(\frac{k}{2}\right)^{m}\right|<\left|a_{m_{i}}\left(\frac{k}{2}\right)^{m_{i}}\right| \text {, for } m<m_{i}
$$

Therefore for $|x|>k$, we have

$$
\left|a_{m}\left(\frac{x}{2}\right)^{m}\right|<\left|a_{m i}\left(\frac{x}{2}\right)^{m_{i}}\right| \text {, for } m<m_{i} .
$$

Now for $|x|>k$,

$$
\left|S_{m_{i}}(x)\right|=\left|\sum_{n=0}^{m_{i}} a_{n}\left(\frac{x}{2}\right)^{n} 2^{n_{i}}\right|=\left|a_{m_{i}}\left(\frac{x}{2}\right)^{m_{i}}\right|\left|2^{m_{i}}+\frac{a_{m_{i}-1}\left(\frac{x^{2}}{2}\right)^{m_{i}-1}}{a_{m_{i}}\left(\frac{x}{2}\right)^{m_{i}}} 2^{m_{i-1}}+\ldots\right| \text {. }
$$

Since $\left|\frac{a_{m-1}\left(\frac{x}{2}\right)^{m_{i-1}}}{a_{m_{i}}\left(\frac{x}{2}\right)^{m_{i}}}\right|<1$, we get

$$
\begin{aligned}
\left|S_{m_{i}}(x)\right| \geqq\left|a_{m i}\left(\frac{x}{2}\right)^{m_{i}}\right|\left(2^{m_{i}}-2^{m_{i}-1}-\ldots-2-1\right) \geqq \\
\qquad\left|a_{m_{i}}\left(\frac{k}{2}\right)^{m_{i}}\right|\left(2^{m_{i}}-\ldots-2-1\right)=\left|a_{m_{i}}\left(\frac{k}{2}\right)^{m_{i}}\right|
\end{aligned}
$$

Consequently $\quad \lim _{i=\infty}\left|S_{m i}(x)\right|=\infty$ uniformly for $|x|>k$.

Theorem V. ${ }^{i=\infty}$ (Kakeya)

Let a power series $\sum_{n=0}^{\infty} \alpha_{n} x^{n}$ have $\alpha$ convergence circle $|x|=r$ and $\rho_{n}$ be 
the maximum modulus of roots of the $n$-th section. Then we have $r \leqq \lim _{n=\infty} \rho_{n} \leqq 2 r$

We may suppose that $a_{0}$ is not zero; Let the roots of the $n$-tin section be denoted by $\alpha_{1}, \ldots \alpha_{n b}$; then

$$
\left|\frac{a_{2}}{a_{n}}\right|=\left|\alpha_{1} \ldots \alpha_{n b}\right| \leqq \rho_{n}^{n}
$$

Hence

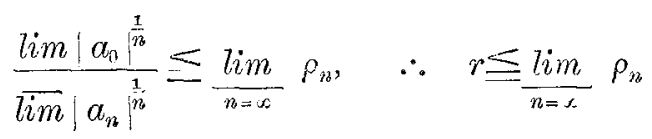

Next suppose if possible that $\frac{\lim }{\bar{n}_{\infty} \infty} \rho_{i b}>2 r$, and let $k$ so taken that $\frac{\lim }{n=\infty} \rho_{i n}$ $>k>2 r$, then for sufficiently large $m, \sum_{n=0}^{n} a_{n} x^{n}=0$ would have a root, whose modulus is greater than $k$ in contradiction to Theorem IV; hence we have $\lim _{n=\infty} \rho_{n} \leqq 2 r$

Remark. Okada's theorem is a special case of Theorem $\mathrm{V}$, when $r=\infty$.

12. We know that $\rho_{n}$ tends to infinity by Okada's theorem. In the following lines $I$ will investigate into the order of $\rho_{n}$ with respect to $n$.

Theorem VI. Let $F(x)=a_{0}+a_{1} x+\ldots+a_{13} x^{n}+\ldots$ be a transcendental integral function, and suppose that for non-vanishing $a_{n}$

$$
\begin{aligned}
& \varlimsup_{l_{b=\infty}}\left|a_{n}\right| n^{k n+r}=K<\infty \\
& \lim _{n=\infty}\left|a_{n}\right| n^{k n+r}=L>0
\end{aligned}
$$

where $k$ is positive and $r$ is any real number.

For any given small quanlity $\epsilon$, we have for sufficiently large $n$

$$
1-\epsilon<\frac{\rho_{n}}{n^{k}}<e^{\frac{\kappa}{L}+k}+\varepsilon
$$

where $\rho_{n}$ is the maximum modulus of roots of the n-th section.

Proof. By the hypothesis we can find $m$ so that for $n>m$,

$$
\frac{L-\varepsilon}{n^{n k+r}} \leqq\left|a_{n}\right| \leqq \frac{K+\varepsilon}{r^{n k+r}}
$$

for non-vanishing $a_{n}$, when $\varepsilon$ is given.

Now put

$$
\left|a_{0}\right|+\left|a_{1}\right|+\ldots+\left|a_{m-1}\right|=M
$$


then for $|x|>1$, we have

$$
\left|a_{3}+a_{1} x+\ldots+a_{m-1} x^{m-1}\right| \leqq M\left|x^{m}\right|
$$

From (64) we get

$$
\left|a_{m} x^{m}+\ldots+a_{n-1} x^{n-1}\right| \leqq(K+\xi)\left[\frac{|x|^{n}}{m^{m k+r}}+\ldots+\frac{|x|^{n-1}}{(n-1)^{(n-1) k+r}}\right]
$$

In order to evaluate the right hand side of $(66)$, we put

$$
f(t)=:-\frac{\mid x_{\mid}^{t}}{t^{t+r}}
$$

Then

$$
f^{\prime}(t)=f(t)\left[\log |x|-k \log t-k-\frac{r}{t}\right]
$$

Take $|x|$ so large, that when $t$ varies from 1 to $n$,

$$
\log |x|-k \log t-k-\frac{r}{t} \geqq \lambda>1
$$

or

$$
|x| \geqq e^{i+\lambda} \cdot t^{k} e^{\frac{r}{t}}
$$

Now we will determine the maximum value of $t^{r} e^{\frac{r}{t}}$ when $t$ varies from 1 to $n$. For this purpose we put

$$
\varphi(t)=t^{\hbar} e^{\frac{r}{t}}
$$

Then

$$
\varphi^{\prime}(t)=e^{\frac{r}{t}} t^{k-2}(k t-r)
$$

Hence $\varphi^{\prime}(t)=0$ gives $t=\frac{r}{k}$, which corresponds to the minimum of $\varphi(t)$.

Hence $\mathcal{\rho}(t)$ has no maximum, consequently it atains the greatest value at $t=1$ or $t=n$ in the interval $(1, n)$.

Since $\varphi(\mathbf{l})=e, r^{r}$ while $\varphi(n)=n^{n} e^{\frac{r}{n}}$ tends to infinity, when $t$ tends to infinity, we see that for a sufficiently large $n, \phi(n)=n^{k} e^{\frac{r}{n}}$ is the greatest value of $\varphi(t)$ in the interval $(1, n)$

From (70) we know that if we take 


$$
|x| \geqq e^{k+\lambda+\frac{r}{16} n^{k}}
$$

then $f(t)$ is increasing in the interval $(1, n)$, we get

$$
\frac{|x|^{m}}{m^{m \mid t+r}}+\ldots+\frac{|x|^{\mid n-1}}{(n-1)^{(n-1) k+r}}<\int_{m}^{n} \frac{|x|^{t} d t}{t^{t k+r}}=\int_{m}^{n} f(t) d t
$$

From (68) and (69)

$$
f^{\prime}(t) \geqq \lambda f(t)
$$

Hence

$$
\int_{m}^{n} f(t) d t \leqq \frac{1}{\lambda}\left[f(t)_{-m}^{\eta^{n}}<\frac{f(n)}{\lambda}=\frac{1}{\lambda} \frac{|x|^{n}}{n^{n^{2}+r}+r} .\right.
$$

From (73) and (7t) we get

$$
\frac{|x|^{m}}{m^{m k+r}}+\ldots+\frac{|x|^{n-1}}{(n-1)^{(n-1) k+\gamma}}<\frac{1}{\lambda} \frac{|x|^{m}}{n^{n k+r}}
$$

From (65) and (75) we have,

$$
\begin{aligned}
\left|a_{3}+a_{1} x+\ldots+a_{n-1} x^{n-1}\right| & <M|x|^{m}+\frac{(K+\varepsilon) \mid x_{\mid}^{n}}{\lambda n^{k n_{3}+r}}= \\
& \frac{(K+\varepsilon)^{\prime}|x|^{n}}{\lambda n^{k n+r}}\left[1+\frac{M \lambda}{K+\varepsilon} \frac{n^{k n+r}}{|x|^{n_{3}-n_{n}}}\right]
\end{aligned}
$$

We will show that $\frac{n^{k_{1 / b+r}}}{|x|^{n-m}}$ can be made ve $y$ small by taking $n$ very large. From (72)

$$
|x| \geqq e^{k+\lambda+\frac{r}{n}} n^{k}>e^{k+\frac{r}{n}} n^{k}
$$

Hence

$$
\frac{n^{n k i+r}}{|x|^{n-m}}<\frac{n^{k m m}}{e^{k i n} e^{-k m+\frac{r}{n}(n-m)}}
$$

Since $\lim _{n=\infty} \frac{n^{a}}{e^{\beta n}}=0$, when $\beta$ is positive, we can determine $n_{v}$ so large that for $n \geq n_{0}$ we have,

$$
\frac{n^{k i m}}{e^{k i n-k m+\frac{r}{n}} \cdot(n-m)}<\varepsilon_{1} \frac{K+\varepsilon}{M}
$$


Hence

$$
\frac{M n^{k n+\cdot r}}{(K+\varepsilon)|x|^{n-m}}<\varepsilon_{1}
$$

Therefore for $n \geqq n$, and $|x| \geqq e^{k+\lambda+\frac{r}{n}} n^{k}$

$$
\left|a_{3}+a_{1} x+\ldots+a_{n-1} x^{n-1}\right|<\frac{|x|^{n}(K+\xi)}{\lambda n^{k n+r}}\left(1+\Sigma_{1} \lambda\right)
$$

Next we will determine $\lambda$, which is not specified till now, so that

$$
L-\varepsilon \equiv \frac{(K+\varepsilon)\left(1+\varepsilon_{1} \lambda\right)}{\lambda}
$$

or

$$
\lambda \equiv \frac{K+\varepsilon}{L-\varepsilon-\varepsilon_{1}(K+\varepsilon)}
$$

This is always possible by taking $\varepsilon_{1}$ very small.

Then

$$
\begin{aligned}
\left|a_{n} x^{n}\right| & \geqq \frac{(L-\varepsilon)|x|^{n}}{n^{k n+r}} \geqq \frac{(K+\varepsilon)\left(1+\varepsilon_{1} \lambda\right)}{\lambda} \frac{|x|^{n}}{n^{k n+r}} \\
& >\left|a_{0}+a_{1} x+\ldots+a_{n-1} x^{n-1}\right|
\end{aligned}
$$

Hence $a_{0}+a_{1} x+\ldots+a_{n} x^{n}=0$ has no root, whose modulus is greater than $n^{k} e^{k+\lambda+\frac{r}{n}}$, hence we have,

$$
\varlimsup_{n=\infty} \frac{\left|x_{n}\right|}{n^{k}} \leqq e^{i+k}
$$

Since $\varepsilon$ and $\varepsilon_{1}$ are arbitrary, we get from (80)

$$
\varlimsup_{n=\infty} \frac{\left|x_{n}\right|}{n^{k}} \leqq e^{\frac{\kappa}{L}+k}
$$

Next let $\alpha_{1} \ldots \alpha_{n}$ be the roots of $a_{0}+a_{1} x+\ldots+a_{n} x^{n}=0$

Then

$$
\left|\alpha_{1} \ldots \alpha_{n}\right|=\left|\frac{a_{0}}{a_{n}}\right|
$$

Hence if we denote the maximum modulus of the roots of the $n$-th section by $\rho_{n}$ we get, 


$$
\left|\frac{a_{n}}{a_{n}}\right| \fallingdotseq \rho_{n}^{n}
$$

Or

Hence

$$
\frac{n^{n+r}+r}{(K+\varepsilon)}\left|a_{0}\right|<\rho_{n}^{n} \quad(n \geqq m)
$$

$$
\lim _{n=\infty} \frac{\rho_{n}}{n^{k}} \geqq 1
$$

The theorem is proved.

Cor. In a transcendental integral function, $F(x)=\sum_{n=0}^{\infty} a_{n} x^{n}$ if for non-vanish ing $a_{n}$

$$
\lim _{n \rightarrow \infty}\left|a_{n}\right| n^{k n+r}=A(\neq 0)
$$

where $k$ is positive and $r$ is any real number, then, when $s$ is given, however small, we can find $n_{0}$ such that for $n \geqq n_{0}$

$$
1-\varepsilon<\frac{\rho_{n}}{n^{k}}<e^{1+k}+-\varepsilon
$$

13. In place of the above hypothesis, we suppose that for nonvanishing $a_{n}$ 。

$$
\begin{aligned}
& \varlimsup_{\substack{n=\alpha \\
n=\alpha}}\left|a_{n}\right|(n !)^{k}=K<\infty \\
& \prod_{n=\infty}\left|a_{n}\right|(n !)^{k}=L>0
\end{aligned}
$$

By Stirling's formula

$$
(n !)^{k}=\left(\frac{n}{e}\right)^{k n}(2 \pi n)^{\frac{k}{2}}\left(1+\frac{k}{n}+\ldots\right) .
$$

Now we put

$$
z=e^{x_{1}} x,
$$

Then

$$
a_{3}+a_{1} x+\ldots+a_{n} x^{n}+\ldots=b_{3}+b_{1} z+\ldots+b_{n} z^{n}+\ldots
$$

where

$$
a_{n}=b_{n_{0}} e^{k r_{n}} \text {. }
$$

Hence

$$
\left|a_{n}\right|(n \mid)^{k}=\left|b_{n}\right| n^{k n+\frac{k}{2}}(2 \pi)^{\frac{k}{2}}\left(1+\frac{k}{n}+\ldots\right) .
$$


Consequently

$$
\begin{aligned}
& \varlimsup_{n=\infty}\left|b_{n}\right| n^{k n+\frac{k}{2}}=K_{1}=\frac{K}{(2 \pi)^{k}} \\
& \frac{\lim _{n=\infty}}{n=b_{2 b} \mid n^{k n+\frac{k}{2}}}=L_{1}=\frac{L}{(2 \pi)^{k}}
\end{aligned}
$$

Hence by Theorem VI we get

$$
1 \leqq \lim _{n=\infty} \frac{\left|z_{i b}\right|}{n^{k}} \leqq \varlimsup_{n=\infty} \frac{\left|\psi_{n b}\right|}{n^{k}} \leqq e^{\frac{\pi_{1}}{L_{1}}+k}=e^{\frac{\kappa}{L}+k}
$$

where $\left|z_{n}\right|$ is the maximum modulus of roots of the $n$-th section:

$$
b_{9}+b_{1} z+\ldots+b_{n} z^{n}=0 \text {. }
$$

From (83) we get

$$
\frac{1}{e^{k}} \leqq \frac{\lim }{n=\infty} \frac{\left|x_{n}\right|}{n^{k}} \leqq \overline{l i m}_{n=\infty} \frac{\left|x_{n}\right|}{n^{k}} \leqq e^{\frac{K}{L}}
$$

Hence we have

Theorem VII. In a transcentental integral function $F(x)=\sum_{n=0}^{\infty} a_{n} x^{n}$ suppose that for non-vanishing $a_{n}$

$$
\begin{aligned}
& \varlimsup_{n=\infty}\left|a_{n}\right|(n !)^{k}=K<\infty \\
& \prod_{n=\infty}\left|a_{n}\right|(n !)^{k}=L>0
\end{aligned}
$$

then for $n \geqq n_{0}$

$$
\frac{1}{e^{k}}-\hat{\varepsilon}<\frac{\rho_{n}}{n^{k}}<e^{\frac{K}{L}}+\varepsilon
$$

when $\varepsilon$ is given.

Cor. In a transcendental integral function $F(x)=\sum_{n=0}^{\infty} a_{n} x^{n}$

if for non-vanisting $a_{n} ; \lim _{n=\infty}\left|\alpha_{n}\right|(n !)^{k}=A(\neq 0)$, then for a sufficiently large $n$,

$$
\frac{1}{e^{k}}-\varepsilon<\frac{\rho_{n}}{n^{k}}<e+\varepsilon
$$

'T'o this cathegory of functions belong $e^{x}, \sin x, \cos x, \sinh x, \cosh x$.

14. Take as another example the Bessel-functions.

$$
J_{r}(z)=\left(\frac{z}{2}\right)^{r} \sum_{n=0}^{\infty} \frac{(-1)^{n}}{\Gamma^{\prime}(n+1) \Gamma(n+r+1)}\left(\frac{z}{2}\right)^{2 n}
$$


The root of sections of $\left(86^{2}\right)$ is equivalent to that of the power series;

$$
F(x)=\sum_{n=0}^{\infty} \frac{(-)^{n}}{I^{\prime}(n+1) \Gamma^{\prime}(n+r+1)} x^{n n}=\sum_{n=0}^{\infty} a_{n} x^{n}
$$

Hence

$$
\begin{aligned}
& z^{2}=4 x \\
& \left|a_{n}\right|=\frac{1}{\Gamma^{\prime}(n+1) L^{\prime}(n+r+1)}
\end{aligned}
$$

By Stirling's formula, we get,

$$
\begin{array}{ll}
\Gamma(n+1)=\left(\frac{n}{e}\right)^{n} \sqrt{2 \pi n}\left(1+s_{n}\right) & \lim \hat{\varepsilon}_{n b}=0 \\
\Gamma(n+r+1)=\left(\frac{n+r}{e}\right)^{n+r} \sqrt{2 \pi n}\left(1+\varepsilon_{n}^{\prime}\right) & \text { lim } \hat{\varepsilon}_{n}{ }^{\prime}=0
\end{array}
$$

Hence

$$
\begin{gathered}
I^{\prime}(n+1) I^{\prime}(n+r+1)=2 \pi \frac{n^{2 n+r+1}}{e^{2 n+r}}\left(1+\frac{r}{n}\right)^{n+r}\left(1+\varepsilon_{n b}^{\prime \prime}\right)= \\
\frac{2 \pi n^{2 n+r+1}}{e^{2 n+r}}\left(1+\frac{r}{n}\right)^{\frac{n}{r} r+r}\left(1+\varepsilon^{\prime \prime}{ }_{13}\right)
\end{gathered}
$$

Since

$$
\lim _{n=\infty}\left(1+\frac{r}{n}\right)^{\frac{n n}{r}}=e
$$

We get

$$
I^{\prime}(n+1) \Gamma^{\prime}(n+r+1)=\frac{2 \pi n^{2 n+r+1}+1}{e^{2 n}}\left(1+\hat{s}_{n}^{\prime \prime \prime}\right)
$$

Hence

$$
\left|a_{n}\right|=\frac{e^{2 n}}{2 \pi n^{2 n+r+1}}\left(1+\varepsilon_{0}\right) \quad \text { Tim } \varepsilon_{0}=0
$$

Next put

Then

$$
y=e^{2} x
$$

$$
\sum_{n=0}^{\infty} a_{n} x^{n}=\sum_{n=0}^{\infty} b_{n} y^{n}
$$

where 
Hence

$$
a_{n}=b_{n} e^{2 n}
$$

$$
\lim _{v_{b}=\infty}\left|b_{n}\right| n^{2 n+r+1}=\frac{1}{2 \pi}
$$

From the above corollary, we get

$$
1 \leqq \lim _{n=\infty} \frac{\left|y_{n}\right|}{n^{2}} \leqq \lim _{n=\infty} \frac{\left|y_{n}\right|}{n^{2}} \leqq e^{3}
$$

where $\left|y_{n}\right|$ is the maximum modulus of roots of the $n$-th section.

From (88) and (9l) we have, $\sqrt{y}=\frac{e z}{2}$

Hence

$$
\frac{2}{e} \leqq \lim _{n=\infty} \frac{\left|z_{n}\right|}{n} \leqq \lim _{n=\infty} \frac{\left|z_{n}\right|}{n} \leqq 2 \sqrt{e}
$$

Theorem VIII. Let $\rho_{n}$ be the maximum modu'us of rocts of the $n$-th section of Bessel-function $\boldsymbol{J}_{\boldsymbol{r}}(\mathrm{a})$, then for a sufficiently large ne wave,

$$
\frac{2}{e}-\varepsilon<\frac{\rho_{n}}{n}<2 \sqrt{e}+\varepsilon
$$

I will here express my cordial thanks to Prof. Kakeya, who gave me the proofs of his theorems which are unpublished and permitted me to publish them in my paper.

Tokyo, Nov. 1924. 